\section{Original Article \\ Pediatrics}

Check for updates

\section{OPEN ACCESS}

Received: Sep 8, 2018

Accepted: Oct 31, 2018

Address for Correspondence:

Dong In Suh, MD, PhD

Department of Pediatrics, Seoul National

University College of Medicine, 101 Daehak-ro,

Jongno-gu, Seoul 03080, Korea.

E-mail: dongins0@snu.ac.kr

C 2019 The Korean Academy of Medical

Sciences.

This is an Open Access article distributed under the terms of the Creative Commons Attribution Non-Commercial License (https:// creativecommons.org/licenses/by-nc/4.0/) which permits unrestricted non-commercial use, distribution, and reproduction in any medium, provided the original work is properly cited.

ORCID iDs

Yun Jung Choi (D)

https://orcid.org/0000-0002-0078-8374 Gwang Cheon Jang (D)

https://orcid.org/0000-0002-2423-9951

Hyeon-Jong Yang (iD

https://orcid.org/0000-0002-7287-4300

Hyo-Bin Kim (D)

https://orcid.org/0000-0002-1928-722X

Young Yoo (iD)

https://orcid.org/0000-0003-3354-6969

Meeyong Shin (D)

https://orcid.org/0000-0003-3699-8741

So-Yeon Lee (iD)

https://orcid.org/0000-0002-2499-0702

Jakyoung Kim (iD)

https://orcid.org/0000-0001-6724-3400

Woo Kyung Kim (D)

https://orcid.org/0000-0001-8730-010X Dong In Suh (D)

https://orcid.org/0000-0002-7817-8728

\title{
The Korean Version of the Test for Respiratory and Asthma Control in Kids (TRACK): Reliability and Validity
}

 \\ Young Yoo $\mathbb{D}^{1},{ }^{6}$ Meeyong Shin $\left(\mathbb{1},{ }^{7}\right.$ So-Yeon Lee $\mathbb{D}^{1},{ }^{8}$ Jakyoung Kim $\mathbb{D},{ }^{9}$ \\ Woo Kyung Kim $\left({ }^{10},{ }^{10}\right.$ Dong In Suh $\left(\mathbb{D},{ }^{1}\right.$ Young Yull Koh', and the Korean childhood \\ Asthma RESearch (KAERS) Group
}

\begin{abstract}
'Department of Pediatrics, Seoul National University College of Medicine, Seoul, Korea ${ }^{2}$ Sowha Children's Hospital, Seoul, Korea

${ }^{3}$ Department of Pediatrics, National Health Insurance Service Ilsan Hospital, Goyang, Korea

${ }^{4}$ Department of Pediatrics, Soonchunhyang University Seoul Hospital, Soonchunhyang University College of Medicine, Seoul, Korea

${ }^{5}$ Department of Pediatrics, Inje University Sanggye Paik Hospital, Inje University College of Medicine, Seoul, Korea

${ }^{6}$ Department of Pediatrics, Korea University College of Medicine, Anam Hospital, Seoul, Korea

${ }^{7}$ Department of Pediatrics, Soonchunhyang University Bucheon Hospital, Soonchunhyang University College of Medicine, Bucheon, Korea

${ }^{8}$ Department of Pediatrics, Asan Medical Center, University of Ulsan College of Medicine, Seoul, Korea

${ }^{9}$ Department of Pediatrics, Kangwon National University Hospital, Chuncheon, Korea

${ }^{10}$ Department of Pediatrics, Inje University Seoul Paik Hospital, Inje University College of Medicine, Seoul, Korea
\end{abstract}

\section{ABSTRACT}

Background: Test for Respiratory and Asthma Control in Kids (TRACK) questionnaires were developed and validated in various languages to monitor respiratory control in preschoolaged children. We aimed to assess the reliability and validity of the Korean version of the TRACK questionnaire.

Methods: We administered the linguistically validated TRACK questionnaires to caregivers of asthmatic preschool children on two separate visits 4-6 weeks apart. Each physician graded the level of the guideline-based asthma control, assessed the timing of symptoms, and adjusted the therapeutic level at each visit.

Results: A total of 137 children were enrolled in the study. Cronbach's alpha was 0.65 for a questionnaire as a whole. The test-retest reliability was 0.72 . The median TRACK scores were significantly different between asthma control status categories, with the lowest scores in children classified as poorly controlled and the highest in the well-controlled group $(P<0.001)$. They were different among groups classified according to the physician adjusted therapeutic levels, with the lowest values in children prescribed step-up therapy $(P<0.001)$, and according to the recency of respiratory symptoms $(P<0.001)$. Finally, the changes in TRACK scores between visits were highest in subjects showing improved control, followed by unchanged, and worsened control. When we applied the traditional cut-off of 80 for a well-controlled condition, a sensitivity of $75.6 \%$ and a specificity of $70.9 \%$ were calculated.

Conclusion: The Korean translated version of the TRACK questionnaire is valid and reliable to assess respiratory and asthma control in Korean preschool children with asthma symptoms.

Keywords: Asthma; Pediatric; Preschool Children; Questionnaire; Validation Studies 
Disclosure

The authors have no potential conflicts of interest to disclose.

Author Contributions

Conceptualization: Yoo Y, Suh DI, Koh YY. Data curation: Choi YJ, Jang GC, Yang HJ, Kim HB, Yoo Y, Shin M, Lee SY, Kim J. Formal analysis: Choi YJ, Yang HJ, Kim WK, Suh DI. Writing original draft: Choi YJ, Kim HB, Suh DI. Writing - review \& editing: Jang GC, Shin M, Lee SY, Kim J, Kim WK, Suh DI, Koh YY.

\section{INTRODUCTION}

Asthma is the most common chronic childhood disease and the leading cause of childhood morbidity resulting in unscheduled medical utilization, emergency department visits, and hospitalizations. ${ }^{1}$ To maintain good asthma control, an accurate assessment of the airway control status is particularly important. ${ }^{2}$ Questionnaires have been utilized as essential tools in assessing asthma control. With the aid of information obtained directly from the caregiver or patient, physicians can evaluate the level of asthma control more efficiently, and then determine treatment level for the upcoming months. ${ }^{3}$ Various asthma questionnaires have been developed for and applied to groups of adults, teenagers, and school-aged children. - $-6^{-}$

For children aged 4 to 11 years, a validated questionnaire is utilized in clinical practice. ${ }^{7-11}$ Both the children and their caregivers complete the questionnaire items together. For children younger than 5 years, a caregiver-based questionnaire, the Test for Respiratory and Asthma Control in Kids (TRACK),11,12 has been developed and validated in several languages. ${ }^{13-15}$ The Turkish and Spanish versions of the TRACK questionnaire, as well as the English version, accurately and reliably reflect asthma control in preschool-aged children.

The TRACK questionnaire has also been translated into Korean and has recently been linguistically validated ${ }^{16}$; however, the accuracy and reproducibility has not yet been verified. In order to utilize the Korean version of the TRACK questionnaire in clinical practice and to collaborate with global research groups, it must first be determined that the Korean version is as valid and reproducible as the original English version.

In this study, we aimed to evaluate the reliability and validity of the Korean version of the TRACK questionnaire. We administered this questionnaire to caregivers of children with asthma symptoms during our regular clinical practice and assessed the reproducibility and clinical validity.

\section{METHODS}

\section{Study population and design}

Study participants were recruited from 8 pediatric allergy clinics from the Korean Childhood Asthma Research (KAERS) Group. Caregivers of asthmatic children younger than 5 years were invited to participate in the study from August 2016 to July 2017. Children were eligible if they met the following criteria: 1 ) a history of 2 or more episodes of wheezing, shortness of breath, or a recurrent cough that lasted more than 24 hours, and 2) either a previous asthma diagnosis by a specialist or an improvement in respiratory symptoms with aerosolized bronchodilator use. Children were excluded if they had respiratory conditions other than asthma that might affect their cardiopulmonary status (e.g., chronic lung disease or congenital heart disease).

\section{TRACK questionnaire}

The original TRACK questionnaire was developed in English to assess and monitor respiratory symptom control in younger children. ${ }^{11}$ It is a 5 -item questionnaire: 4 questions evaluate the impairment, and the other assesses the risk of asthma control. Each question within the table was scored on a 5-point scale, from 0 to 20, with a total score ranging from 
0 to 100 . High TRACK scores indicate that subjects have better respiratory control, and a score of less than 80 suggests poor control,11,12 which is well-replicated in other language versions of the TRACK questionnaire.13,14 To test the reliability and validity, we adopted the Korean version of the TRACK questionnaire that was validated linguistically in a previous study through a multistep process of forward-translation, reconciliation, back-translation, cognitive debriefing, and proofreading. ${ }^{16}$

\section{Study design and procedures}

During the study period, all caregivers visited each center at least two times, as an initial (baseline) visit and a follow-up visit occurring 4-6 weeks apart. At the baseline visit, we collected demographic data from children and their accompanying caregivers. We administered the questionnaires to caregivers before meeting with physicians on every visit; by doing so, the caregiver responses would be blinded to each physician. During the patient encounter, each physician assessed the currentness of symptoms, graded the level of respiratory control based on the National Asthma Education and Prevention Program Expert Panel Report (NAEPP EPR) 3 guideline recommendations, ${ }^{3}$ and adjusted the therapeutic level at each visit. Children's respiratory symptoms were classified into 3 categories according to the presence or persistence of wheezing, shortness of breath, or a recurrent cough: currently symptomatic (episodes in the previous 4 weeks and in the previous 12 months), symptomatic in the recent past (episodes in the past 12 months, but not within the past 4 weeks), or asymptomatic (no episode for more than 12 months). Asthma control was also graded as one of the following three categories: well-controlled, not well-controlled, or poorly controlled. Finally, therapeutic levels were adjusted to either a step-up in therapy, no change, or a stepdown in therapy.

\section{Reliability}

We assessed the internal consistency of the Korean version questionnaire by evaluating the Cronbach's alpha for the total items. The test-retest reliability was assessed through the intra-class correlation (ICC) between TRACK scores at the baseline and follow-up visits in a subgroup of patients whose control levels were well-controlled on both visits. ${ }^{17}$

\section{Validity}

Construct validity was assessed using the known-groups validity approach. ${ }^{18}$ To assess the discriminant validity, we compared baseline TRACK scores across the three asthma control categories. We also compared the TRACK scores across the three categories of therapeutic adjustment and the currentness of respiratory symptoms at the baseline visit. To assess TRACK's sensitivity to change in respiratory control, we compared the changes in TRACK scores across the three groups in whom the level of control was improved, unchanged, or worsened between the initial and follow-up visits. The receiver operating characteristic (ROC) curve analyses were applied to TRACK scores at baseline and follow-up visits to determine the cut-off TRACK scores for differentiating subjects who are in good respiratory control from other subjects.

\section{Statistical analysis}

We adopted the one-way ANOVA or Kruskal-Wallis non-parametric method to compare TRACK scores across groups as appropriate. The cut-off points for discriminating between well-controlled groups and the others were set using the ROC curve analysis. Statistical analysis was done with the $\mathrm{R}$ statistic software (version 3.3.2; R Foundation for Statistical Computing, Vienna, Austria), and a statistical significance level was set at $P<0.05$. 


\section{Ethics statement}

The current study was approved by the Institutional Review Boards (IRBs) of Seoul National University Hospital (1507-134-689), National Health Insurance Service Ilsan Hospital (NHIMC-2016-10-005), Soonchunhyang University Seoul Hospital (SCHUH 2015-12-005007), Inje University Sanggye Paik Hospital (SGPAIK 2015-10-007), Korea University Anam Hospital (2016AN0305), Soonchunhyang University Bucheon Hospital (SCHBC 2015-09011), Hallym University Sacred Heart Hospital (2015-1119), and Kangwon National University Hospital (2016-04-007). Informed permission was obtained but assent was waived.

\section{RESULTS}

\section{Patient population}

A total of 137 children (67.2\% male) participated in the study. The demographic characteristics of patients and caregivers are listed in Table 1 . The mean \pm standard deviation (SD) age of the patients was $3.5 \pm 1.1$ years and that of the caregivers was $36.9 \pm 7.6$ years. Most $(91.2 \%)$ caregivers were the patients' mothers and $40.2 \%$ of subjects exhibited wellcontrolled status.

\section{Reliability}

Cronbach's alpha was 0.65 at baseline and 0.68 at the follow-up visit for the questionnaire as a whole. When the fifth item was excluded from the analysis, the Cronbach's alpha for baseline visits increased to 0.72 . The intraclass correlation coefficient for test-retest reliability was 0.72 (95\% confidence interval [CI], 0.48-0.85, $P<0.001)$ for a subgroup of children whose guideline-based levels of control were well-controlled at both visits $(\mathrm{n}=42)$.

Table 1. Demographic characteristics of patients and caregivers $(n=137)$

\begin{tabular}{lc}
\hline Characteristics & Values \\
\hline Child & \\
$\quad$ Male & $92(67.2)$ \\
Age, yr & $3.5 \pm 1.1$ \\
Age groups, yr & \\
$\quad 0-1$ & $14(10.2)$ \\
$2-3$ & $77(56.2)$ \\
$\quad 4-5$ & $46(33.6)$ \\
Caregiver & \\
Age, yr & $36.9 \pm 7.6$ \\
Relation & $125(91.2)$ \\
$\quad$ Mother & $4(3.0)$ \\
$\quad$ Father & $8(5.8)$ \\
$\quad$ Grandparents & \\
Education & $6(4.4)$ \\
$\quad$ High school graduate or under & $64(46.7)$ \\
$\quad$ College graduate & $33(24.1)$ \\
$\quad$ Graduate school or higher & $34(24.8)$ \\
$\quad$ Unidentified & \\
Disease control rating & $54(39.4)$ \\
Not well-controlled & $28(20.4)$ \\
Poorly controlled & $55(40.2)$ \\
Well-controlled & \\
\hline
\end{tabular}

Values are presented as number (\%) or mean \pm standard deviation. 
Table 2. Comparison of TRACK scores among subject groups classified by the physician's rating of the level of control and their adjustment in the level

\begin{tabular}{|c|c|c|c|c|c|c|c|c|}
\hline \multirow[t]{2}{*}{ TRACK score } & \multicolumn{4}{|c|}{ Physicians' rating on children's level of control } & \multicolumn{4}{|c|}{ Physicians' adjustment in the treatment level } \\
\hline & Well-controlled & Not well-controlled & Poorly controlled & $P$ value & Step down & No change & Step up & $P$ value \\
\hline \multirow{2}{*}{$\begin{array}{l}\text { Baseline TRACK score, } \\
\text { median (IQR) }\end{array}$} & $\mathrm{n}=55$ & $\mathrm{n}=54$ & $\mathrm{n}=28$ & $<0.001$ & $\mathrm{n}=22$ & $\mathrm{n}=71$ & $\mathrm{n}=44$ & $<0.001$ \\
\hline & $85.0(75.0-95.0)$ & $70.0(55.0-80.0)$ & $55.0(42.5-67.5)$ & & $92.5(81.5-100.0)$ & $75.0(60.0-90.0)$ & $65.0(50.0-75.0)$ & \\
\hline \multirow{2}{*}{$\begin{array}{l}\text { Follow-up TRACK score, } \\
\text { median (IQR) }\end{array}$} & $\mathrm{n}=89$ & $n=34$ & $\mathrm{n}=8$ & $<0.001$ & $n=18$ & $n=103$ & $n=10$ & $<0.001$ \\
\hline & $90.0(80.0-95.0)$ & $75.0(60.0-85.0)$ & $60.0(45.0-67.5)$ & & $97.5(87.5-100.0)$ & $80.0(70.0-90.0)$ & $57.5(35.0-75.0)$ & \\
\hline
\end{tabular}

TRACK = Test for Respiratory and Asthma Control in Kids, IQR = interquartile range.

Table 3. Comparison of TRACK scores according to the currentness of respiratory symptoms at the baseline visit

\begin{tabular}{|c|c|c|c|c|}
\hline & \multicolumn{4}{|c|}{ Currentness of respiratory symptoms } \\
\hline & Current $(n=63)$ & Recent-past $(n=38)$ & Asymptomatic $(n=36)$ & $P$ value \\
\hline TRACK score, median (IQR) & $65.0(47.5-75.0)$ & $72.5(65.0-85.0)$ & $92.5(80.0-100.0)$ & $<0.001$ \\
\hline
\end{tabular}

TRACK = Test for Respiratory and Asthma Control in Kids, IQR = interquartile range.

\section{Validity}

The median TRACK scores across the subject groups concerning the levels of respiratory control and the therapeutic-plan adjustment are listed in Table 2. The median TRACK scores differed significantly among the well-controlled, not well-controlled, and the poorly controlled groups ( $P<0.001$ at both visits), with the highest value reflected in the wellcontrolled group (median, 85.0; interquartile range [IQR], 75.0-95.0) and the lowest in the poorly controlled group (median, 55.0; IQR, 42.5-67.5) at the baseline visit. At the follow-up visit, a similar trend was observed across the subject groups $(P<0.001)$.

On the other hand, TRACK scores differed significantly across the groups categorized by the physicians' therapeutic plan adjustment (i.e., step-down, no change, and step-up) $(P<0.001$ at both visits), with the highest value reflected in the step-down group (median, 92.5; IQR, 81.5-100.0) and the lowest in the step-up group (median, 65.0; IQR, 50.0-75.0) at the baseline visit. At the follow-up visit, a similar trend was observed across the treatment level modifications $(P<0.001)$.

Table 3 shows the baseline TRACK score of subject groups according to the currentness of respiratory symptoms. There was a significant difference in TRACK scores $(P<0.001)$; those who had current asthma symptoms presented the lowest TRACK scores (median, 65.0; IQR, 47.5-75.0) and those who did not have asthma symptoms within the past 12 months had the highest TRACK scores (median, 92.5; IQR, 80.0-100.0).

\section{Responsiveness}

The responsiveness of the TRACK questionnaire to the changes in respiratory condition has also been evaluated: the difference in the TRACK score changes from baseline to followup visit is depicted in Fig. 1. Among the three categories of condition changes (improved, unchanged, and worsened) TRACK scores differed significantly $(P<0.001)$, with the highest, positive values reflected by those who had improved condition (median, 15.0; IQR, 5.0-25.0), and the lowest, negative values by those who had a worsened respiratory condition (median, -10.0 ; IQR, -22.5, -7.5).

\section{ROC curve analysis}

We calculated the best cut-off for differentiating subjects with a well-controlled condition from the not-well-controlled or poorly controlled conditions (Fig. 2). At the baseline visit, TRACK scores of 75 displayed the best sensitivity and specificity $(64.6 \%$ and $83.6 \%$, 


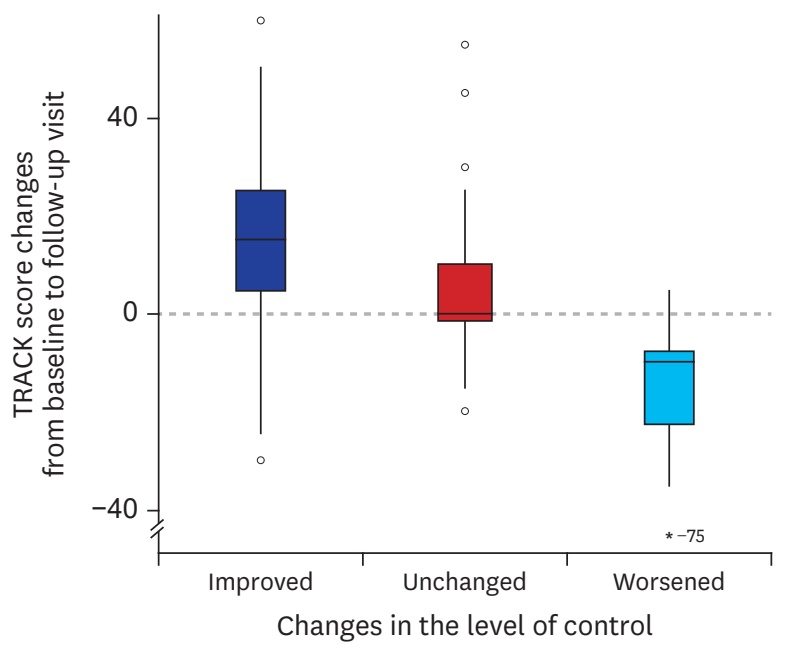

Fig. 1. The difference in the TRACK score changes from baseline to follow-up visit. Box and whisker plot showing the median alteration in TRACK score from baseline to follow-up visits among children in each of the three control-level change categories.

TRACK = Test for Respiratory and Asthma Control in Kids.

outlier value; *extreme outlier value.

respectively) with an area under the curve (AUC) of 0.83. At the follow-up visit, TRACK scores of 80 were suggested from the ROC curve analysis as the best cut-off for differentiating subjects with well-controlled respiratory condition (sensitivity $69.0 \%$; specificity $75.3 \%$; and an AUC of 0.76; IQR, 0.66-0.85; $P<0.001$, respectively; figures not suggested). When we apply the traditional cut-off of 80 from the various versions of TRACK questionnaires to the baseline visit TRACK scores, a sensitivity of $75.6 \%$ and a specificity of $70.9 \%$ were calculated from the ROC curve analysis.

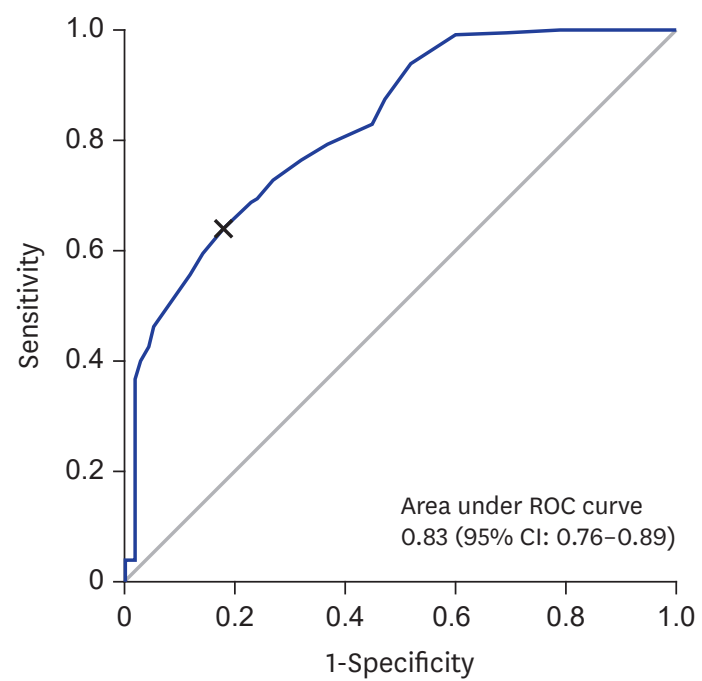

\begin{tabular}{lccccc}
\hline TRACK score & Sensitivity & Specificity & PPV & NPV & Accuracy \\
\hline$<75$ & $64.6 \%$ & $83.6 \%$ & $85.5 \%$ & $61.3 \%$ & $72.3 \%$ \\
$<80$ & $75.6 \%$ & $70.9 \%$ & $79.5 \%$ & $66.1 \%$ & $73.7 \%$ \\
\hline
\end{tabular}

Fig. 2. ROC curve for TRACK score cutoff in differentiating well-controlled children from the others. $\mathrm{ROC}=$ receiver operating characteristic, TRACK $=$ Test for Respiratory and Asthma Control in Kids, $\mathrm{Cl}=\mathrm{confidence}$ interval, PPV = positive predictive value, NPV = negative predictive value. 


\section{DISCUSSION}

In this study, we assessed the reliability and validity of the Korean version of the TRACK questionnaire in Korean children younger than 5 with asthma symptoms. The Cronbach's alpha for the total items, and the intraclass correlation of the TRACK scores of both visits, were at an acceptable level. TRACK scores were different between groups when we classified subject groups according to the level of asthma control, the modification of therapeutic plans, the timing of asthma symptoms, and the change in control levels. ROC curve analysis showed that the cut-off point of 80 , as suggested by previous studies for the baseline visit, has good sensitivity and specificity.

We found the Korean version of the TRACK questionnaire to be reliable and reproducible. The Cronbach's alpha values at baseline and follow-up visits were 0.65 and 0.68 respectively, which were below the accepted level of $0.70 .{ }^{19}$ But the value of the baseline visit increased to 0.72 when we excluded the fifth item of the TRACK questionnaire from the analysis. This finding is similar to that of the previous study, ${ }^{12}$ where the Cronbach's alpha values for the original version were 0.68 at the baseline and 0.64 at the follow-up visit. Those values were increased to 0.76 at the baseline visit, when the fifth item was excluded from the analysis. These findings imply that the initial 4 items in the questionnaire reflect the same aspects of asthma control, and the other fifth item represents another element, which corresponds with the impairment and the risk domain of asthma control, respectively. As for the reproducibility, we evaluated the test-retest reliability and found a good ICC value of $0.72 .{ }^{20}$ We confined the analysis only to well-controlled subjects at both visits, because the variation within the control status is minimal in well-controlled subjects in comparison with not wellcontrolled or poorly controlled subjects.

The TRACK scores showed significant differences among the control levels, which is the primary finding of our study. The original version of the TRACK questionnaire and other language versions ${ }^{12-14}$ consistently shown a significant difference between groups: the highest level in subjects of the well-controlled group and the lowest in the poorly controlled group. These results indicate that the items of the TRACK questionnaire reflect the essential determinant of asthma control, regardless of language or country. On the other hand, the TRACK scores were different between groups classified according to therapeutic plan: the highest level in subjects that needed the step-up therapy and the lowest in those who could afford the step-down treatment. Similarly, the currentness of symptoms is also reflected in the TRACK score. Those subjects who presented current respiratory symptoms had the lowest TRACK scores. These findings are in accordance with the previous findings, 12-14 and suggest that the Korean TRACK questionnaire has adequate validity. Moreover, these findings imply that the Korean version may also be applied to global studies comparing treatment outcomes, as well as to clinical practice.

The TRACK questionnaire also reflected changes in the patients' asthma conditions. The change in TRACK scores between the two visits was significantly different among groups when we classified the subjects according to changes in their levels of respiratory control: those who presented an improved level of respiratory control, (i.e., from poorly controlled to not-well controlled or well-controlled, and from not-well controlled to well-controlled) showed the highest TRACK score changes. On the other hand, those who showed worsened respiratory control displayed a negative value and the lowest TRACK score changes. The similar trend between the change in control status and that in the TRACK score may 
indicate that the TRACK score is a sensitive tool for reflecting the change in subjects' respiratory control.

One of the additional benefits of using the asthma questionnaire is that the patients or caregivers who complete the questionnaire can proactively understand their asthma conditions. By setting up the cut-off point, the patients/caregivers may cooperate with physicians to keep their asthma in good control. Previous TRACK questionnaires in various language versions have suggested cut-off scores of 80 to screen subjects out of the "well-controlled" group and allow them to consult with their physicians. ${ }^{11,13,14}$ In our study, the ROC curve indicated 75 and 80 as the best cut-off for differentiating well-controlled subjects from the others at the baseline and follow-up visits, respectively. This inconsistency in the best cut-off TRACK scores between visits has already been shown in the validation study of the original TRACK questionnaire, which calculated the best cut-off as 75 and 85 for the baseline and follow-up visits, respectively. ${ }^{12}$ Therefore we evaluated the sensitivity and specificity of the cut-off point of 80 , a universally accepted cut-off determined by the various versions of TRACK questionnaires instead, and we found a sensitivity of $75.6 \%$ and a specificity level of $70.9 \%$, which we consider acceptable to screen asthmatic subjects who need to consult with their physicians.

In this study, there was a discrepancy between the level of control and the therapeutic plan, which indicates that subjects with well-controlled asthma received either a step-down therapy or no change in their treatment, whereas physicians recommended either a step-up therapy or no change to subjects with not well-controlled asthma. A recent report found that Korean pediatric asthma specialists frequently modify prescriptions based on their individual decisions, rather than following the international guidelines unconditionally. ${ }^{21}$ Therefore, the TRACK score should not be interpreted as a need to change their therapeutic plans; rather it should only be interpreted concerning a need to consult with their physicians.

There are two ways of adopting the asthma questionnaire: to translate an existing foreign questionnaire, or to create a new one. A Japanese pediatric society recently built a new questionnaire, the Best Asthma Control Test for Preschoolers (BEST ACT-P), for children aged $<6$ years. They weighed the virtue of Japanese-specific optimization for younger Japanese children. ${ }^{22}$ However, our study focused more on the universal adaptability that enables us to collaborate internationally. Additionally, we already completed the linguistic validation of the Korean translated TRACK questionnaire, ${ }^{16}$ which enhanced the feasibility of developing a Korean asthma questionnaire through a translation for younger children. Although the TRACK questionnaire is no longer the only questionnaire suitable for subjects $<5$ years, this study would give more credit to the TRACK questionnaire as a standard tool for assessing respiratory control in younger children aged $<5$ years.

Our study has enrolled a relatively small number of patients compared to the number of centers involved. Since we gathered a minimum number of variables, it may not be possible to adjust variation across centers. Although the physicians who participated in this study were national board-certified allergy specialists, there might be individual variation in determining the level of treatment across centers. Another limitation of our study is the issue of asthma diagnosis. We enrolled subjects with physician-diagnosed asthma, or recurrent wheezing with symptomatic bronchodilator responsiveness, which may not coincide with the classical diagnostic criteria within the older age groups. Finally, environmental or background factors (i.e., caregiver opinions or changes in the season or weather) might have affected the results. 
In conclusion, the Korean translated version of the TRACK questionnaire has adequate reliability, validity, and responsiveness. This questionnaire may enable us to evaluate the level of asthma control more efficiently and reliably in our daily practice or global studies.

\section{ACKNOWLEDGMENTS}

AstraZeneca, the owner of the original version of the TRACK questionnaire, permitted us to translate and validate the questionnaire.

\section{REFERENCES}

1. Bahadori K, Doyle-Waters MM, Marra C, Lynd L, Alasaly K, Swiston J, et al. Economic burden of asthma: a systematic review. BMC Pulm Med 2009;9(1):24. PUBMED | CROSSREF

2. Reddel HK, Taylor DR, Bateman ED, Boulet LP, Boushey HA, Busse WW, et al. An official American Thoracic Society/European Respiratory Society statement: asthma control and exacerbations: standardizing endpoints for clinical asthma trials and clinical practice. Am J Respir Crit Care Med 2009;180(1):59-99. PUBMED | CROSSREF

3. National Asthma Education and Prevention Program. Expert Panel Report 3 (EPR-3): Guidelines for the Diagnosis and Management of Asthma-Summary Report 2007. J Allergy Clin Immunol 2007;120(5 Suppl):S94-138. PUBMED | CROSSREF

4. Cloutier MM, Schatz M, Castro M, Clark N, Kelly HW, Mangione-Smith R, et al. Asthma outcomes: composite scores of asthma control. J Allergy Clin Immunol 2012;129(3 Suppl):S24-33. PUBMED | CROSSREF

5. Kwon HS, Lee SH, Yang MS, Lee SM, Kim SH, Kim DI, et al. Correlation between the Korean version of Asthma Control Test and health-related quality of life in adult asthmatics. J Korean Med Sci 2008;23(4):621-7. PUBMED | CROSSREF

6. Kim HS, Kim YH, Lee HS, Han YK, Park YA, Kim KW, et al. Utility of tools for the assessment of asthma control in childhood asthma. Allergy Asthma Respir Dis 2015;3(4):261-6. CROSSREF

7. Juniper EF, Gruffydd-Jones K, Ward S, Svensson K. Asthma Control Questionnaire in children: validation, measurement properties, interpretation. Eur Respir J 2010;36(6):1410-6. PUBMED | CROSSREF

8. Liu AH, Zeiger R, Sorkness C, Mahr T, Ostrom N, Burgess S, et al. Development and cross-sectional validation of the Childhood Asthma Control Test. J Allergy Clin Immunol 2007;119(4):817-25. PUBMED | CROSSREF

9. Schatz M, Sorkness CA, Li JT, Marcus P, Murray JJ, Nathan RA, et al. Asthma Control Test: reliability, validity, and responsiveness in patients not previously followed by asthma specialists. J Allergy Clin Immunol 2006;117(3):549-56. PUBMED | CROSSREF

10. Vollmer WM, Markson LE, O'Connor E, Sanocki LL, Fitterman L, Berger M, et al. Association of asthma control with health care utilization and quality of life. Am J Respir Crit Care Med 1999;160(5 Pt 1):1647-52. PUBMED | CROSSREF

11. Murphy KR, Zeiger RS, Kosinski M, Chipps B, Mellon M, Schatz M, et al. Test for Respiratory and Asthma Control in Kids (TRACK): a caregiver-completed questionnaire for preschool-aged children. J Allergy Clin Immunol 2009;123(4):833-9.e9. PUBMED | CROSSREF

12. Chipps B, Zeiger RS, Murphy K, Mellon M, Schatz M, Kosinski M, et al. Longitudinal validation of the Test for Respiratory and Asthma Control in Kids in pediatric practices. Pediatrics 2011;127(3):e737-47. PUBMED | CROSSREF

13. Rodríguez-Martínez CE, Nino G, Castro-Rodriguez JA. Validation of the Spanish version of the Test for Respiratory and Asthma Control in Kids (TRACK) in a population of Hispanic preschoolers. J Allergy Clin Immunol Pract 2014;2(3):326-31.e3. PUBMED | CROSSREF 
14. Buyuktiryaki B, Sahiner UM, Yavuz ST, Cavkaytar O, Arik Yilmaz E, Soyer OU, et al. Validation of the Turkish version of "Test for Respiratory and Asthma Control in Kids (TRACK)" questionnaire. J Asthma 2013;50(10):1096-101. PUBMED | CROSSREF

15. Zeiger RS, Mellon M, Chipps B, Murphy KR, Schatz M, Kosinski M, et al. Test for Respiratory and Asthma Control in Kids (TRACK): clinically meaningful changes in score. J Allergy Clin Immunol 2011;128(5):983-8. PUBMED | CROSSREF

16. Oh HL, Koh YY, Suh DI, Kang BC, Kim BS, Kim WK, et al. Translation and linguistic validation of Korean version of the Test for Respiratory and Asthma Control in Kids instrument. Allergy Asthma Respir Dis 2016;4(1):22-30. CROSSREF

17. Weir JP. Quantifying test-retest reliability using the intraclass correlation coefficient and the SEM.J Strength Cond Res 2005;19(1):231-40. PUBMED | CROSSREF

18. Davidson M. Known-groups validity. In: Michalos AC, editor. Encyclopedia of Quality of Life and Well-Being Research. Dordrecht: Springer; 2014, 3481-2. CROSSREF

19. Nunnally JC, Bernstein IH. Psychometric Theory. 3rd ed. New York, NY: McGraw-Hill; 1994.

20. Streiner DL, Norman GR. Health Measurement Scales: a Practical Guide to Their Development and Use. 2nd ed. Oxford: Oxford University Press; 1995.

CROSSREF

21. Suh DI, Yang HJ, Kim BS, Shin YH, Lee SY, Park G, et al. Asthma severity and the controller prescription in children at 12 tertiary hospitals. Allergy Asthma Immunol Res 2017;9(1):52-60. PUBMED | CROSSREF

22. Sato K, Sato Y, Nagao M, Shimojo N, Yoshihara S, Adachi Y, et al. Development and validation of asthma questionnaire for assessing and achieving best control in preschool-age children. Pediatr Allergy Immunol 2016;27(3):307-12.

PUBMED | CROSSREF 